\title{
W-Plasty Scar Revision of Broad Facial Scars
}

\author{
HELMY EL-WAKEEL, M.D.; EHAB Z. AZZAM, M.D. and AHMED A. ZZEDAN, M.D. \\ The Department of Plastic Surgery, Faculty of Medicine, Alexandria University, Egypt
}

\begin{abstract}
Background: The decision to revise a scar should be based on risk versus benefit. Broad scars usually could be improved with scar revision. In the face typically those broad scar run perpendicular to RSTLs which is a well-known predisposing factor for a broad scar deformity. Scar irrgularization techniques as W-plasty is an effective technique to improve and camouflage such broad scars.
\end{abstract}

Material and Methods: W-plasty scar revision was employed for repair of old broad facial scars in 13 patients, scars were more than one year old.

Results: Significant improvement was obtained in all patients based on objective assessment of scar improvement at 6 month post-operative, using visual analog scale (VAS). All patients rated their improvements as excellent, good or acceptable.

Conclusion: W-plasty scar revision yielded highly acceptable cosmetic outcomes in patients suffering from broad facial scars running away from RSTLs.

Key Words: W-plasty - Scar revision - RSTLs.

\section{INTRODUCTION}

Facial scars usually results from trauma, their location, orientation and length is quiet variable. The severity of scar deformity depend upon many factors including the severity and nature of injury, the depth of wound, condition of skin edges and possible skin loss which would affects not only the appearance of the scar but also the extent of distortion of adjacent structures. Other factors affecting the degree scaring include, the relation of the scar to relaxed skin tension lines and facial landmarks, patient age, genetic factors, and the quality of wound repair [1-3].

An ideal scar is fine, flat line, matching the color of the surrounding skin, this is typically seen in scars oriented along the relaxed skin tension lines (RSTLs) [2,3]. Scar revision procedures are designed to optimize the appearance of potentially correctable scar deformity. This could be limited by the location, shape and size of the scar, surrounding facial landmarks, and patient genetics [3].
During patient consultation about scar revision, it is important to emphasize that the procedure aim and capability is just improving the scar not erasing it; as a miss concept of having an invisible scar is common among patients seeking scar revision.

A variety of surgical techniques are described for a scar revision, this include simple fusiform excision and repair, serial scar excision, Z-plasty, scar irregularization techniques as W-plasty and geometric broken line closures and more complex revisions with local flaps, and tissue expansion for more complex scars with tissue loss or distortion [1-7].

An ugly scar may be too wide, contracted, atrophic, pigmented, depressed, raised, or causing distortion of a facial landmark. The choice of the most suitable scar revision technique for a particular scar, depends upon the nature of the scar deformity presented and surgeon experience.

Scar irregularization with W-plasty is reported to offer good scar camouflage in widened and contracted scars that typically run away from relaxed skin tension lines [2-7].

Aim: To study the efficacy of W-plasty scar revision for improving broad facial scars oriented away from relaxed skin tension lines.

\section{PATIENTS AND METHODS}

Between April, 2016 to July, 2018 a total of 13 patients with old broad facial scars were enrolled in this study.

\section{Inclusion criteria:}

- Patients having broad facial scars oriented away from relaxed skin tension lines.

- Patients having realistic expectations.

- Scars of 1 year or more old. 
Pre-operative:

During preoperative patients counseling, patients expectations were brought to a realistic level, informed consent and photographs were taken.

Design (Fig. 1):

W-plasty design was done before LA injection using fine surgical marking pen. A series of consecutive triangles were outlined, on one side of a scar and its mirror image on the other side of the scar. The arms of the $\mathrm{W}$ were about $4-6 \mathrm{~mm}$ in length and angled between 60-90 degrees depending on the relationship of the scar to the RSTLs. One limb of the triangle was drawn parallel to the RSTLs.

Hand free drawing was used; aiming for some degree of dissimilarity between consecutive triangular flaps, in a trial for overcoming the main disadvantage of W-plasty of creating a regularly irregular scar that some what noticeable.

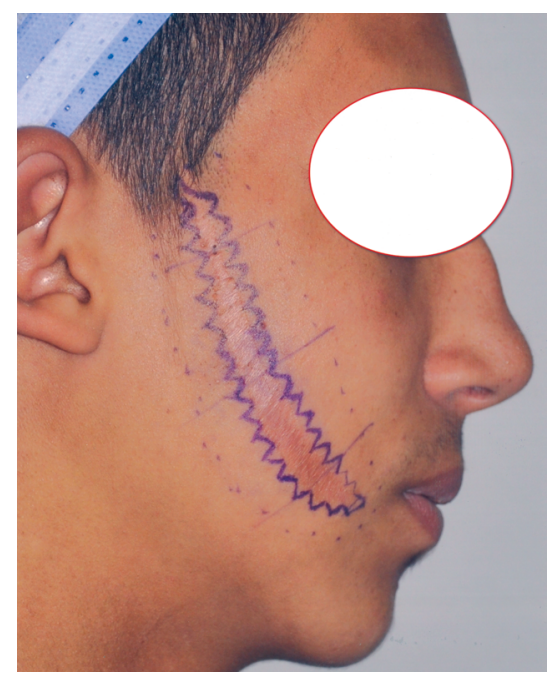

Fig. (1): W-plasty design.

\section{Anesthesia:}

All patients were operated under local anesthesia with 1: 100,000 epinephrine.

\section{Surgical technique:}

Skin incision was made perpendicular to the skin surface using 11 blade. The original scar and attached triangular pieces of normal skin were excised and the skin edges were judiciously undermined using a No. 15 blade, in the subdermal fat layer above the SMAS. The extent of undermining ranged from 1$2 \mathrm{~cm}$ around the incision to permit tension free closure. Bleeding was controlled by compression and judicious use of bipolar cautery. Atraumatic tissue handling using fine toothed tissue forceps and skin hooks with minimal manipulation of wound margins was the role. Wound margins were kept moist with a moist sponge during the procedure.
Wound closure was done by interdigitating the triangular flaps in a single zigzag line. Closure of the subcutaneous layer was the initial step with buried simple interrupted deep dermal sutures using 5/0 PDS suture (Ethicon, Inc., Somerville, NJ); this served the functions of everting the epidermis, decreasing dead space, and provided tension free skin closure.

Final skin closure was done using simple interrupted 6/0 Prolene suture (Ethicon, Inc., Somerville, NJ), then Steri-Strips (3M, St. Paul, MN) was placed for added protection and support.

\section{Post-operative care and follow-up:}

Skin sutures were removed after 5 days and strapping with Steri-Strips was continued for 6 weeks post-operative for added wound protection and support.

A visual analog scale (VAS) $(0-10 \mathrm{~cm} ; 0=$ best, $10=$ worst) was used for scar assessment before and 6 month after revision by 2 independent plastic surgeon not involved in the study, tacking the average of the 2 scores as our reference.

Patient satisfaction was assessed 6 month postoperative and patients were asked to classify their results as either: Excellent, good, acceptable, poor, or very poor.

Possible complications as hypertrophic scaring, or worsening of the scar were noted.

\section{RESULTS}

Study results are summarized in Table (1). Sturdy enrolled 13 male patients with old post traumatic broad facial scars. Mean patient's age was 24.9 years, most frequent scar location was cheeks (9 patients) and average scar length was $7.1 \mathrm{~cm}$.

There were no intraoperative complications or major postoperative complications in any of the patients (including but not limited to clinically significant hematoma, wound dehiscence, skin flap necrosis, or hypertrophic scars).

Objective assessment by VAS showed significant improvement of the scars at 6 month post-operative. The mean of pre-operative VAS score for all patients was (7.8), this turned to (3.3) six months after Wplasty scar revision. Figs. $(2,3,4)$ presents pre and 6 month post-operative results of 3 patients.

All patients were satisfied with their results at 6 months follow-up, with 3 patients (out of 13) rating their improvement as excellent, 8 patients reported good results and 2 patients rated their results as acceptable. 
Table (1): Patient's demographic data and results.

\begin{tabular}{|c|c|c|c|c|c|c|}
\hline $\begin{array}{l}\text { Patient } \\
\text { No. }\end{array}$ & $\begin{array}{l}\text { Age in } \\
\text { years }\end{array}$ & Scar location & $\begin{array}{l}\text { Scar } \\
\text { length }\end{array}$ & $\begin{array}{l}\text { Pre-op } \\
\text { VAS score }\end{array}$ & $\begin{array}{l}\text { Post-op } \\
\text { VAS score }\end{array}$ & $\begin{array}{c}\text { Patient rating } \\
\text { of results }\end{array}$ \\
\hline 1 & 14 & L cheek \& upper lip & $7 \mathrm{~cm}$ & 8 & 2 & Excellent \\
\hline 2 & 19 & $\mathrm{R}$ mandibular border & $6 \mathrm{~cm}$ & 8 & 3 & Good \\
\hline 3 & 43 & L cheek & $11 \mathrm{~cm}$ & 7 & 3 & Good \\
\hline 4 & 33 & R cheek & $7 \mathrm{~cm}$ & 8.5 & 4 & Good \\
\hline 5 & 22 & Forehead & $6 \mathrm{~cm}$ & 7 & 3 & Acceptable \\
\hline 6 & 24 & L cheek & $6 \mathrm{~cm}$ & 8 & 2.5 & Excellent \\
\hline 7 & 28 & $\mathrm{R}$ cheek & $5 \mathrm{~cm}$ & 7.5 & 4 & Good \\
\hline 8 & 20 & $\mathrm{~L}$ preauricular & $5 \mathrm{~cm}$ & 8 & 3 & Good \\
\hline 9 & 18 & $\mathrm{R}$ cheek \& temple & $13 \mathrm{~cm}$ & 9 & 4.5 & Good \\
\hline 10 & 36 & L cheek & $9 \mathrm{~cm}$ & 7.5 & 3 & Good \\
\hline 11 & 27 & Forehead & $5 \mathrm{~cm}$ & 8 & 4 & Good \\
\hline 12 & 21 & L cheek & $7 \mathrm{~cm}$ & 8 & 3 & Excellent \\
\hline 13 & 19 & L cheek & $6 \mathrm{~cm}$ & 7 & 4 & Acceptable \\
\hline Mean & 24.9 & & $7.1 \mathrm{~cm}$ & 7.8 & 3.3 & \\
\hline
\end{tabular}
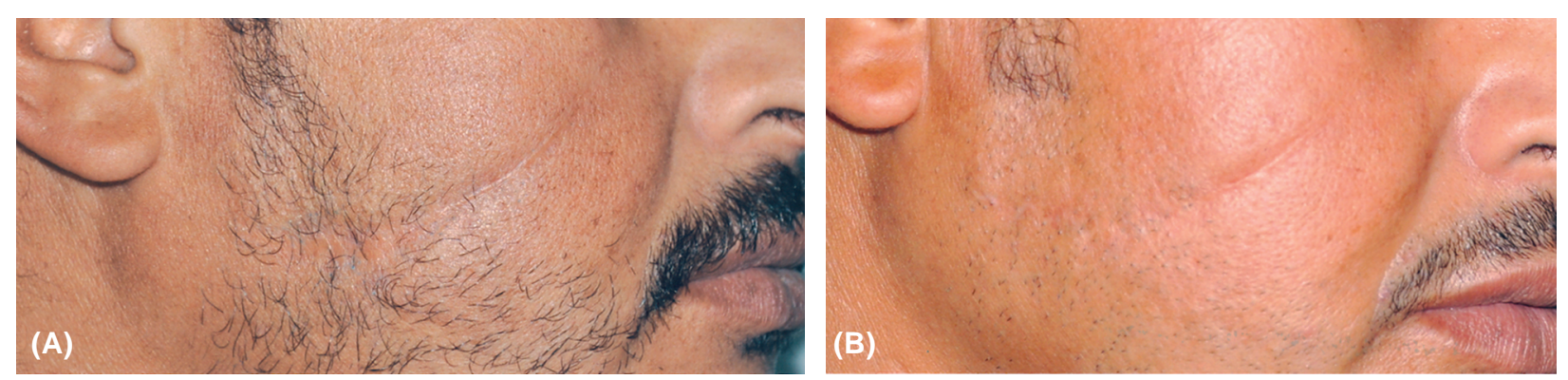

Fig. (2): Pre (A) and 6 months post-operative (B) results of patient No. 7.

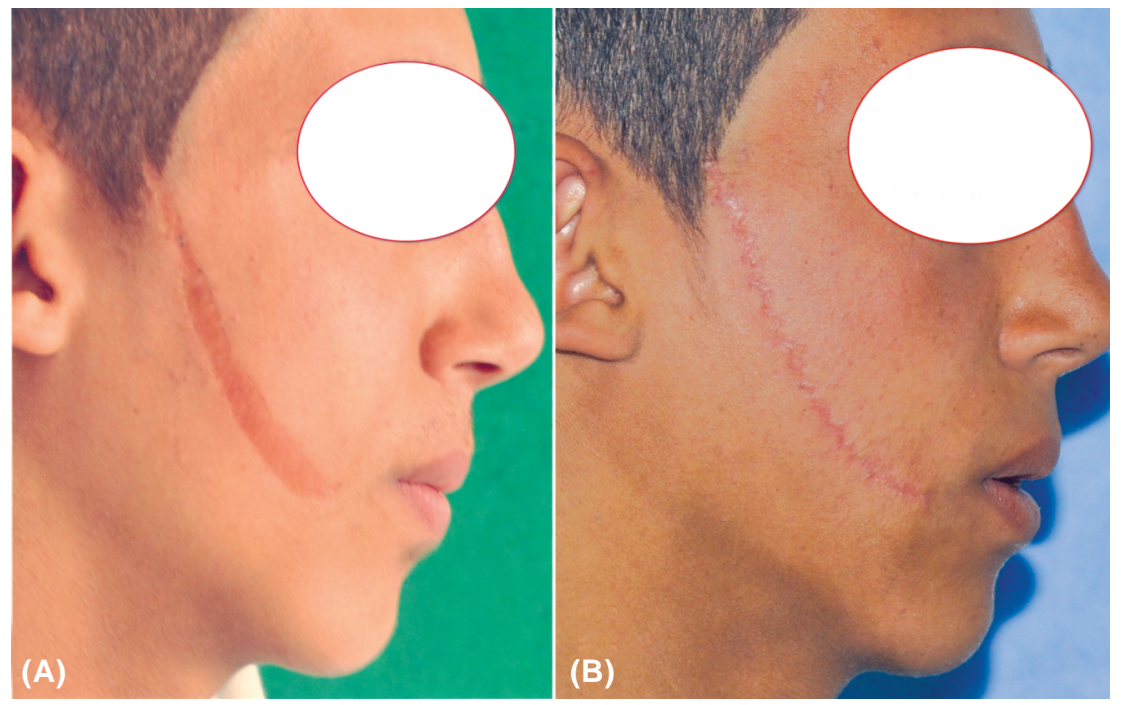

Fig. (3): Pre (A) and 6 months post-operative (B) results of patient No. 9.
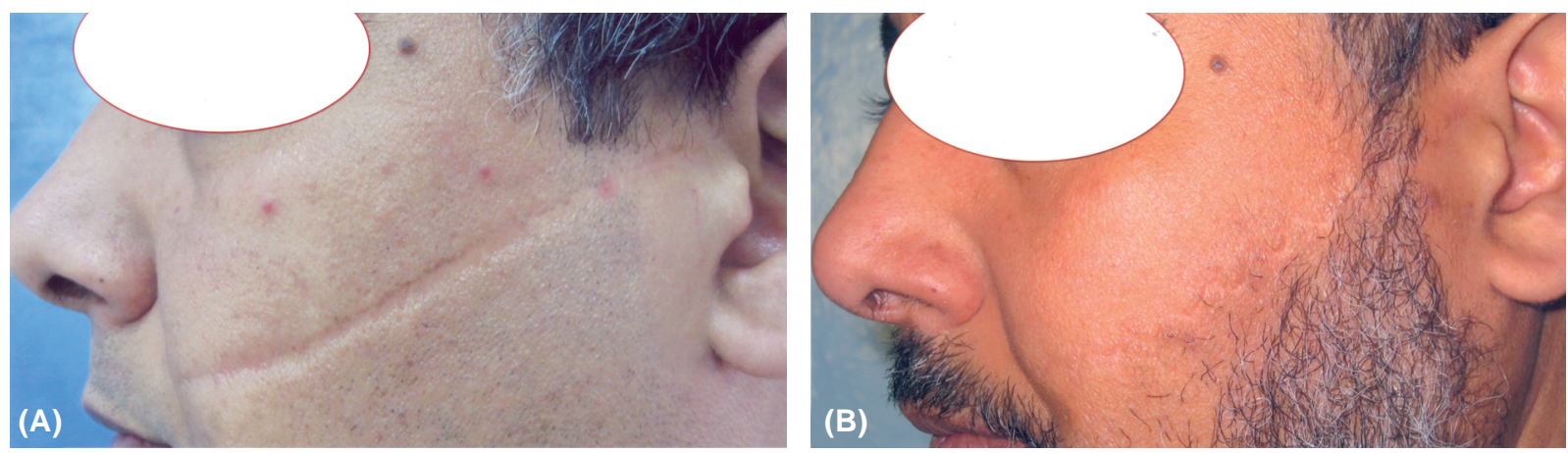

Fig. (4): Pre (A) and 6 months post-operative (B) results of patient No. 3. 


\section{DISCUSSION}

An ideal scar is a fine line that falls within or parallel to a naturally occurring skin creases, or relaxed skin tension line (RSTL). The scar should have the same contour and color of the surrounding skin and does not distort adjacent structures. This should be the goal of scar revision surgery [1-3].

Many techniques are described for a scar revision; of which scar irregularization by W-plasty or geometric broken line closure has been used for scar camouflage for many years, both are especially useful for broad scars that are not oriented parallel to RSTLs. Scar irregularization makes it less noticeable because of insignificant light reflection from irregular scars and it is difficult for the eye to trace and perceive irregular, broken-up scars as compared to linear scars [1-5].

The W-plasty scar irregularization first described by Borges [7] in 1959; consists of multiple small triangular advancement flaps on either sides of the scar such that the closure results in zigzag line so, it breaks a straight scar into multiple small segments, planned to lie parallel to RSTL. Wplasty is easy to plan and execute, it's main disadvantage is that the resultant regular repetitive pattern makes the scar somewhat conspicuous, as the eye easily can follow the predictable regular zigzag configuration [2-4].

Geometric Broken Line Closure is designed to convert a long linear scar into a randomly irregular scar. Interdigitating geometric lines are drawn in such a manner that triangles, rectangles, squares and even semicircles are created on either side of the scar in a random fashion. Majority of the lines should lie along the RSTL. Compared to W-plasty GBLC is a more sophisticated scar irregularization technique than $\mathrm{W}$-plasty, and requires more time

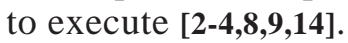

In this study W-plasty triangular flaps of 4$6 \mathrm{~mm}$ long were used, since limbs less than $3 \mathrm{~mm}$ long do not break up the scar adequately and limbs more than $6 \mathrm{~mm}$ long may result in a more conspicuous scar [2-4].

Hand free design of W-plasty triangular flaps was used, aiming for some degree of dissimilarity between consecutive triangular flaps in a trial of overcoming the main disadvantage of W-plasty (compared to GBLC) of creating a regularly irregular scar that somewhat could be traced by the eye. This dissimilarity of triangular flap design was done by allowing a limited variability of flap length between $4-6 \mathrm{~mm}$ and flaps angles in the same pa- tients, but anyway flaps were mirror imaged along scar borders. This little modification from the classic W-plasty design was a trial to capture the advantage of W-plasty of being relatively faster and easier to perform compared to GBLC, while trying to diminish its major disadvantage of a relatively predictable, regularly irregular scar that is somewhat conspicuous.

All of patient enrolled in this study achieved considerable improvement of their scar deformity, a finding that is consistent with literature reports for W-plasty scar revision [2-7,11,12]. Objective assessment by VAS showed significant improvement of the scars at 6 month post-operative. The mean of VAS pre-operative for all patients was (7.8), this turned to be (3.3) at 6 months after Wplasty scar revision.

All patients were satisfied with their results at 6 months follow-up, with 3 patients (out of 13) rating their improvement as excellent, 8 patients reported good results and 2 patients rated their results as acceptable.

\section{Limitations:}

The main shortcoming of this study was lack of compassion to classic regular design W-plasty and GBLC, and of course small sample size. Also, despite using a validated scar evaluation tool (which is photograph-based VAS), the aesthetic outcomes were still based on a subjective assessment of scar appearance.

\section{Conclusion:}

W-plasty yield highly acceptable cosmetic outcomes in patients suffering from broad facial scars running away from RSTLs.

\section{REFERENCES}

1- Shockley W.W.: Scar revision techniques: Z-plasty, Wplasty, and geometric broken line closure. Facial Plast. Surg. Clin. North Am., 19 (3): 455-463, 2011.

2- Watson D. and Reuther M.S.: Scar revision techniquespearls and pitfalls. Facial Plast. Surg., 28: 487-91, 2012.

3- Schweinfurth J.M. and Fedok F.: Avoiding pitfalls and unfavourable outcomes in scar revision. Facial Plast. Surg., 17: 273-8, 2001.

4- Rodgers B.J., Williams E.F. and Hove C.R.: W-plasty and geometric broken line closure. Facial Plast. Surg., 17: 239-44, 2001.

5- Borges A.F.: W-plasty. Ann. Plast. Surg., 3: 153-9, 1979.

6- Borges A.F.: Principles of scar camouflage. Fac. Plast. Surg., 1: 181-190, 1984.

7- Elshahat A1 and Lashin R.: The subunit principle in scar face revision. J. Craniofac. Surg., 28 (4): 1027-1029, 2017. 
8- Alsarraf R. and Murakami C.S.: Geometric broken line closure. Face. Plast. Surg. Clin. North Am., 6: 163-166, 1998.

9- Cupp C.L., et al.: Scar revision-a simplified method to design geometric broken line closures. Fac. Plast. Surg. Clin. North Am., 6: 195-201, 1998.

10- Thomas J.R. and Ehlert T.K.: Scar revision. Fac. Plast. Reconstr. Surg., 5: 45-52, 1992.

11- Jáuregui E.J., Tummala N., Seth R., et al.: Comparison of W-plasty vs traditional straight-line techniques for primary paramedian forehead flap donor site closure. JAMA Facial Plast. Surg., 18 (4): 258-262, 2016.

12- Park S.S.: Scar revision through W-plasty. Fac. Plast. Clin. North Am., 6: 157-161, 1998.

13- Webster R.D., Davidson T.M. and Smith R.C.: Broken line scar revision. Clin. Plast. Surg., 4: 263-274, 1977.

14- Thomas J.R.: Geometric broken-line closure. In: Thomas Jr., Holt G.R., eds. Facial scars: Incisions, revision and Camouflage. St. Louis: CV Mosby, 160-167, 1989. 